\title{
Model for a Universe described by a non-minimally coupled scalar field and interacting dark matter
}

\author{
J. B. Binder*and G. M. Kremer ${ }^{\dagger}$ \\ Departamento de Física, Universidade Federal do Paraná \\ Caixa Postal 19044, 81531-990 Curitiba, Brazil
}

\begin{abstract}
In this work it is investigated the evolution of a Universe where a scalar field, non-minimally coupled to space-time curvature, plays the role of quintessence and drives the Universe to a present accelerated expansion. A non-relativistic dark matter constituent that interacts directly with dark energy is also considered, where the dark matter particle mass is assumed to be proportional to the value of the scalar field. Two models for dark matter pressure are considered: the usual one, pressureless, and another that comes from a thermodynamic theory and relates the pressure with the coupling between the scalar field and the curvature scalar. Although the model has a strong dependence on the initial conditions, it is shown that the mixture consisted of dark components plus baryonic matter and radiation can reproduce the expected red-shift behavior of the deceleration parameter, density parameters and luminosity distance.
\end{abstract}

\section{Introduction}

The measurements of the rotation curves of spiral galaxies [1] as well as other astronomical experiments suggest that the luminous matter represents only a small amount of the massive particles of the Universe, and that the more significant amount is related to dark matter.

Recently the astronomical observations with super-novae of type Ia suggested that our Universe is presently submitted to an accelerated expansion [2] 3]; the nature of the responsible entity, called dark energy, still remains unknown. The simplest explanation for the acceleration is a cosmological constant (see 4]), which fits the present data very well but has some important unsolved problems. Another possibility is to introduce a scalar field $\phi(t)$, which has been extensively studied by the scientific community. By considering a barotropic equation of state for the scalar field $p_{\phi}=\omega \rho_{\phi}$, we have a constant value of $\omega=-1$ for the cosmological constant model and a variable $\omega \geq-1$ for a minimally coupled scalar field model. However, the measured data from Hubble Space Telescope [5] states the restriction for $\omega=-1.02_{-0.19}^{+0.13}$, whereas some recent observations [6] has drawn the attention to the viability of models where $\omega<-1$, which would invalidate both cosmological constant and minimally coupled scalar field descriptions. Some phantom field models were proposed in order to contemplate this hypothesis [7, but they face also some strong difficulties [8].

In this work we consider a scalar field non-minimally coupled to space-time curvature, which was investigated in 9] and widely studied recently, among others, in the works [10, 11, 12, 13, 14]. In a more recent paper 15] the suitability of such models was studied and it was shown that the Einstein field equations are stable under some conditions for the constant that couples the scalar field with the curvature scalar.

Aside several models for the dark sector interaction [16, 17, 18, 19] - that could replace the cosmological constant model in the case of future experimental tests - we consider here a direct

\footnotetext{
*jbb01@fisica.ufpr.br

${ }^{\dagger}$ kremer@fisica.ufpr.br
} 
coupling between dark matter and dark energy. In this model, studied in [20] and more recently in 21], the dark matter particle mass is proportional to the value of the scalar field that represents the dark energy. However, in this model it is necessary to have some precautions, since by assuming the dark matter particle mass to change with time, we could make the dark matter energy density to become physically inconsistent at early stages. We emphasize that in this work the full set of differential equations are solved numerically, unlike the asymptotic approximation of the work 21. The baryonic matter and radiation are described here as usual non-interacting components, being the first non-relativistic and pressureless. However, for the dark matter two models are considered: the first one, by assuming the dark matter as pressureless, and the second one, by using a thermodynamic theory in order to relate the effects of the non-minimally coupling to the dark matter pressure. All components will be described by a set of field equations, and the resulting observables, - i.e., the density parameters, the decelerating parameter and the luminosity distance - which are obtained as solutions of the field equations will be compared to the available data set in order to drawn the conclusions about the viability of this model. We show that physically acceptable solutions are obtained, if we chose initial conditions restricting the rolling nature of the scalar field at early stages. Furthermore, there exist some freedom parameters that will be important to fit the data from incoming experiments. The work is organized as follows: in section 2 the basic field equations for each component, i.e. dark energy, dark matter, baryons and radiation, are derived. The solutions for the pressureless dark matter are found in section 3 for given initial conditions, coupling and potential constants, whereas in section 4 we give the corresponding solutions for the dark matter with non-vanishing pressure. We close the work with section 5 where we address to some remarks and summarize the results of previous sections. Units have been chosen so that $8 \pi G=c=\hbar=k=1$, whereas the metric tensor has signature $(+,-,-,-)$.

\section{Field equations}

We shall consider that the Universe is modeled as a mixture of a scalar field which plays the role of dark energy, a dark matter field and non-interacting baryons and radiation. Here we adopt a more general scalar field which is non-minimally coupled to curvature [9], and a dark matter field whose particle mass is proportional to the value of the scalar field at each point 21. The action for this model is written as:

$$
S=\int d^{4} x \sqrt{-g}\left\{\frac{1}{2}\left(1-\xi \phi^{2}\right) R+\frac{1}{2} \partial_{\mu} \phi \partial^{\mu} \phi-V(\phi)+\frac{1}{2} \partial_{\mu} \chi \partial^{\mu} \chi-\frac{1}{2} \zeta^{2} \phi^{2} \chi^{2}+\mathcal{L}_{r}+\mathcal{L}_{b}\right\},
$$

where $\phi$ and $\chi$ are the dark energy and dark matter scalar fields, respectively, $V(\phi)$ is the dark energy potential density, $\xi$ is the coupling constant between dark energy and the curvature scalar $R$ and $\zeta \phi$ is the dark matter particle mass, with $\zeta$ being a constant. Furthermore, $\mathcal{L}_{r}$ and $\mathcal{L}_{b}$ denote the lagrangian densities of the radiation and of the baryons, respectively.

By taking the variation of the action (11) with respect to the metric tensor $g_{\mu \nu}$, it follows Einstein field equations

$$
R_{\mu \nu}-\frac{1}{2} R g_{\mu \nu}=-\frac{T_{\mu \nu}}{\left(1-\xi \phi^{2}\right)}
$$

In the above equation the total energy-momentum tensor $T_{\mu \nu}$ of the sources of the gravitational field is a sum of the energy-momentum tensors of the baryons $T_{\mu \nu}^{b}$, radiation $T_{\mu \nu}^{r}$, dark matter $T_{\mu \nu}^{d m}$ and scalar field $T_{\mu \nu}^{\phi}$, i.e., $T_{\mu \nu}=T_{\mu \nu}^{b}+T_{\mu \nu}^{r}+T_{\mu \nu}^{d m}+T_{\mu \nu}^{\phi}$. The expressions for the energy-momentum tensors read

$$
\begin{gathered}
T_{\mu \nu}^{b}=\frac{2}{\sqrt{-g}} \frac{\delta \sqrt{-g} \mathcal{L}_{b}}{\delta g^{\mu \nu}}, \quad T_{\mu \nu}^{r}=\frac{2}{\sqrt{-g}} \frac{\delta \sqrt{-g} \mathcal{L}_{r}}{\delta g^{\mu \nu}} \\
T_{\mu \nu}^{d m}=\partial_{\mu} \chi \partial_{\nu} \chi-\left(\frac{1}{2} \partial_{\sigma} \chi \partial^{\sigma} \chi-\frac{1}{2} \zeta^{2} \phi^{2} \chi^{2}\right) g_{\mu \nu}, \\
T_{\mu \nu}^{\phi}=\partial_{\mu} \phi \partial_{\nu} \phi-\left(\frac{1}{2} \partial_{\sigma} \phi \partial^{\sigma} \phi-V\right) g_{\mu \nu}-\xi\left(\nabla_{\mu} \nabla_{\nu} \phi^{2}-g_{\mu \nu} \nabla_{\sigma} \nabla^{\sigma} \phi^{2}\right) .
\end{gathered}
$$


We call attention to the fact that due to the inclusion of the coupling between the scalar field and the scalar curvature, the covariant divergence of the total energy-momentum tensor does not vanish. Indeed, one can obtain from (2) by using Bianchi identities

$$
\nabla_{\nu} T^{\mu \nu}=2 \xi \phi\left(R^{\mu \nu}-\frac{1}{2} R g^{\mu \nu}\right) \partial_{\nu} \phi,
$$

indicating that the total energy-momentum tensor is not a conserved quantity. However, we shall assume that the covariant divergence of the energy-momentum tensors of the baryons and radiation vanish, i.e., $\nabla_{\nu} T_{b}^{\mu \nu}=0$ and $\nabla_{\nu} T_{r}^{\mu \nu}=0$, since they are considered as non-interacting fields from the decoupling age up to the present time.

The evolution equations for the scalar and dark matter fields are obtained from Euler-Lagrange equations, yielding

$$
\nabla_{\sigma} \nabla^{\sigma} \phi+\frac{d V}{d \phi}+\xi R \phi+\zeta^{2} \phi \chi^{2}=0, \quad \nabla_{\sigma} \nabla^{\sigma} \chi+\zeta^{2} \phi^{2} \chi=0 .
$$

Henceforth, we shall restrict ourselves to the study of the consequences of the above model on a Universe which is isotropic, homogeneous and spatially flat, described by the Robertson-Walker metric:

$$
d s^{2}=d t^{2}-a(t)^{2}\left(d x^{2}+d y^{2}+d z^{2}\right),
$$

where $a(t)$ denotes the cosmic scale factor.

For an isotropic and homogeneous Universe the most general representation for the total energymomentum tensor in a comoving frame is given by $\left(T^{\mu}{ }_{\nu}\right)=\operatorname{diag}(\rho,-p,-p,-p)$, where the total energy density $\rho$ and pressure $p$ of the mixture are given in terms of the respective quantities for its constituents, namely, $\rho=\rho_{b}+\rho_{r}+\rho_{d m}+\rho_{\phi}$ and $p=p_{b}+p_{r}+p_{d m}+p_{\phi}$. From now on, we shall assume that the baryons are non-relativistic particles so that $p_{b}=0$ and that the barotropic equation of state for the radiation field $p_{r}=\rho_{r} / 3$ holds.

Now for the Robertson-Walker metric, Einstein field equations (2) lead to the following modified forms of the Friedmann and acceleration equations

$$
H^{2}=\frac{\rho}{3\left(1-\xi \phi^{2}\right)}, \quad \frac{\ddot{a}}{a}=-\frac{\rho+3 p}{6\left(1-\xi \phi^{2}\right)},
$$

respectively, due to the non-minimally coupling of the scalar field to the gravitational field. Above $H=\dot{a}(t) / a(t)$ is the Hubble parameter and the dot denotes a differentiation with respect to time.

The hypothesis of homogeneity implies that the fields $\phi$ and $\chi$ must be only functions of time so that the evolution equations for the dark energy (17) 1 and dark matter (17) ${ }_{2}$ fields reduce to

$$
\begin{gathered}
\ddot{\phi}+3 H \dot{\phi}+\frac{d V}{d \phi}+\frac{\xi \phi}{1-\xi \phi^{2}}(\rho-3 p)+\frac{1}{\phi}\left(\rho_{d m}-p_{d m}\right)=0, \\
\ddot{\chi}+3 H \dot{\chi}+\zeta^{2} \phi^{2} \chi=0,
\end{gathered}
$$

respectively.

The energy densities and the pressures of the dark energy and dark matter fields can now be determined from (4) and (5), yielding

$$
\begin{gathered}
\rho_{\phi}=\frac{1}{2} \dot{\phi}^{2}+V+6 \xi H \phi \dot{\phi}, \quad p_{\phi}=\frac{1}{2} \dot{\phi}^{2}-V-2 \xi\left(\phi \ddot{\phi}+\dot{\phi}^{2}+2 H \phi \dot{\phi}\right), \\
\rho_{d m}=\frac{1}{2} \dot{\chi}^{2}+\frac{1}{2} \zeta^{2} \chi^{2} \phi^{2}, \quad p_{d m}=\frac{1}{2} \dot{\chi}^{2}-\frac{1}{2} \zeta^{2} \chi^{2} \phi^{2} .
\end{gathered}
$$

The evolution equation for the energy density of the scalar field is obtained by taking the time derivative of its energy density (12) 1 which, after some rearrangements, yields

$$
\dot{\rho}_{\phi}+3 H\left(\rho_{\phi}+p_{\phi}\right)=-\left(\rho_{d m}-p_{d m}\right) \frac{\dot{\phi}}{\phi}-\frac{2 \xi \phi \dot{\phi} \rho}{1-\xi \phi^{2}},
$$


thanks to (10) and (12). Following the same methodology, the time derivative of the energy density of the dark matter [13] 1 leads to the evolution equation for dark matter density:

$$
\dot{\rho}_{d m}+3 H\left(\rho_{d m}+p_{d m}\right)=\left(\rho_{d m}-p_{d m}\right) \frac{\dot{\phi}}{\phi},
$$

by using equations (11) and (13) 2 . From equations (14) and (15) we infer that there exists a transfer of energy from the scalar field to the dark matter field which is given by the term $\left(\rho_{d m}-p_{d m}\right) \dot{\phi} / \phi$. Moreover, the term $2 \xi \phi \dot{\phi} \rho /\left(1-\xi \phi^{2}\right)$ in (14) is the responsible for the energy transfer from the scalar field to the gravitational field.

One can obtain from equation (15) together with the definition $\rho_{d m}=m_{d m} n_{d m}=\zeta \phi n_{d m}$ a general expression for the particle number density for dark matter which reads

$$
\dot{n}_{d m}+3 H n_{d m}=-\frac{p_{d m}}{m_{d m}}\left(3 H+\frac{\dot{\phi}}{\phi}\right) .
$$

The above equation clearly simplifies to the usual $\dot{n}_{d m}+3 H n_{d m}=0$ for the vanishing dark matter pressure case and otherwise leads to a non conserved dark matter particle number density due to the particle creation by thermodynamic processes. In the next sections we shall find the cosmological solutions for this model by considering two cases, namely, a pressureless dark matter and a dark matter field with non-vanishing pressure.

The conservation of the energy-momentum tensor of baryons and radiation with $p_{b}=0$ and $p_{r}=\rho_{r} / 3$ lead to the well-known relationships $\rho_{b} \propto 1 / a^{3}$ and $\rho_{r} \propto 1 / a^{4}$.

\section{Pressureless dark matter}

For the case of a pressureless dark matter we have $p_{d m}=0$ and it follows from equation (16) that $\rho_{d m} \propto \phi / a^{3}$. Moreover, this last relationship implies that the particle number density of the dark matter is conserved, i.e., $\dot{n}_{d m}+3 H n_{d m}=0$. Here we have only one equation to solve - namely, the evolution equation for the scalar field (10) - in order to obtain the time evolution of the acceleration field and of the energy densities of each component. By changing the variables, we can express the equation for the dark energy scalar field (10) in terms of the red-shift $z$, since $a=1 /(1+z)$ by assuming that $a(0)=1$. Hence it follows

$$
\frac{(1+z)^{2} \rho}{3\left(1-\xi \phi^{2}\right)} \phi^{\prime \prime}-\frac{(1+z)(\rho-3 p)}{6\left(1-\xi \phi^{2}\right)} \phi^{\prime}+\frac{d V}{d \phi}+\frac{\xi \phi(\rho-3 p)}{1-\xi \phi^{2}}+\frac{\rho_{d m}}{\phi}=0
$$

where hereafter the prime denotes the differentiation with respect to $z$.

In order to solve (17) we have to choose a form for the potential density. Among the several existent models (see, for example 22]), we select a potential that was also used in the references 20. 21], 23]:

$$
V(\phi)=\frac{K}{\phi^{\alpha}}
$$

where $K$ is a constant. This form has the property of blowing up for small values of $\phi$, preventing the field - and therefore the dark matter energy density - from becoming negative. Furthermore, we have to specify initial conditions for the scalar field and its derivative as well as for the energy densities of the baryons, radiation and dark matter. The initial conditions for the energy densities were chosen from the present known values given in the literature (see 24 for a review) for the density parameters $\Omega_{i}(z)=\rho_{i}(z) / \rho(z)$, i.e., $\Omega_{b}(0)=0.04995, \Omega_{r}(0)=5 \times 10^{-5}$ and $\Omega_{d m}(0)=0.23$. The constant $K$ was determined from the present value for the dark energy density parameter, i.e., $\Omega_{\phi}(0)=0.72$, since it was considered that the present value of the scalar field has a small value, so that $\rho_{\phi}(0) \approx V$. There still remain $\alpha$ and $\xi$ as free parameters, whose influence will be studied later in this section. 
We have plotted in figure 1 a phase-portrait that exhibits the evolution of a set of initial conditions. By varying the values of the scalar field in order to produce small variations in the initial dark energy density, it follows that some solutions evolve into a non-physical behavior since they lead to negative values of $\rho_{\phi}$, whereas there exists another set of solutions that causes the dark energy density to increase too fast and is the responsible for almost all contributions to $H$ and $\rho$. In order to get a physical acceptable behavior, it is necessary to impose a very small and positive value for the scalar field slope at early epochs, i.e., $z \gtrsim 1000$. Physically this means that the scalar field starts to roll more significantly around that time and then evolves until today. Moreover, negative derivative of the scalar field at high red-shifts would imply on negative dark energy density, due to (12).

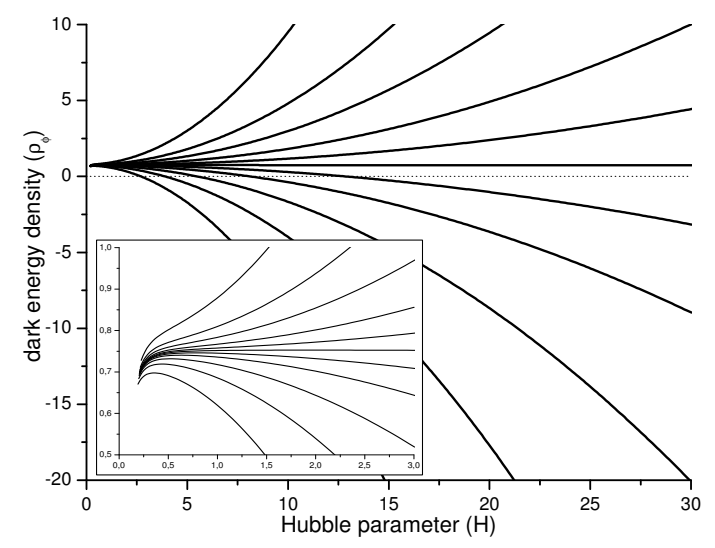

Figure 1: Typical phase-portrait $\left(\rho_{\phi}, H\right)$ corresponding to the case $\alpha=1 / 3$ and $\xi=-0.2$ showing the evolution for both physical $\left(\rho_{\phi}>0\right)$ and non-physical $\left(\rho_{\phi}<0\right)$ solutions.

The red-shift evolution of the density parameters is plotted in the left frame of figure 2 We have considered a fixed value for the exponent $\alpha$, namely $\alpha=1 / 3$, and different values for the coupling constant $\xi$. Two different cases were analyzed: (a) a slightly varying scalar field where the scalar field does not change significantly with the red-shift, showing that the differences from the usual quintessence model $(\xi=0)$ are basically due to the influence of the non-minimal coupling $(\xi=-0.2)$; (b) a strongly varying scalar field, characterized by $\xi=-0.3$, where the initial value of the scalar field has a smaller value than that of the former case but it has a more accentuated change when the red-shift increases. We infer from this figure that by increasing the red-shift the density parameter of the dark energy decays more slowly for the strongly varying case $(\xi=-0.3)$ followed by the slightly varying case when $\xi=-0.2$ and $\xi=0$, whereas the density parameter of the dark matter increases more slowly for these cases. This can be understood by noting that the last term of the right-hand side of equation (14) - that depends on the coupling constant - reduces the energy transfer from the scalar to the dark matter field. For $\xi=0$ the dark energy-dark matter equality occurs at $z \approx 0.4$, the dark energy-baryons equality at $z \approx 1.4$, whereas for $\xi=-0.2$ and $\xi=-0.3$ these equalities occur at higher red-shifts. Moreover, for $\xi=0$ the radiation-matter (baryons plus dark matter) equality occurs at $z \approx 3700$, whereas for $\xi=-0.2$ and $\xi=-0.3$ this equality happens for lower and higher red-shift values, respectively.

The deceleration parameter $q=-\ddot{a} a / \dot{a}^{2}$ as a function of the red-shift $z$ is plotted in the right frame of figure2 We note that by increasing the coupling constant the transition from a decelerated to an accelerated phase occurs at lower red-shifts. According to present experimental values [5] the decelerated-accelerated transition occurs at $z_{T}=0.46 \pm 0.13$ and we can observe from figure 2 that for the slightly varying case with $\xi=-0.2$ this transition is at $z_{T}=0.46$, whereas for the strongly varying case with $\xi=-0.3$ at $z_{T}=0.43$. However, in both cases the present value of the deceleration parameter has a smaller modulus than the one given in the literature for the $\Lambda C D M$ case, i.e., $q_{0} \approx-0.55$. 

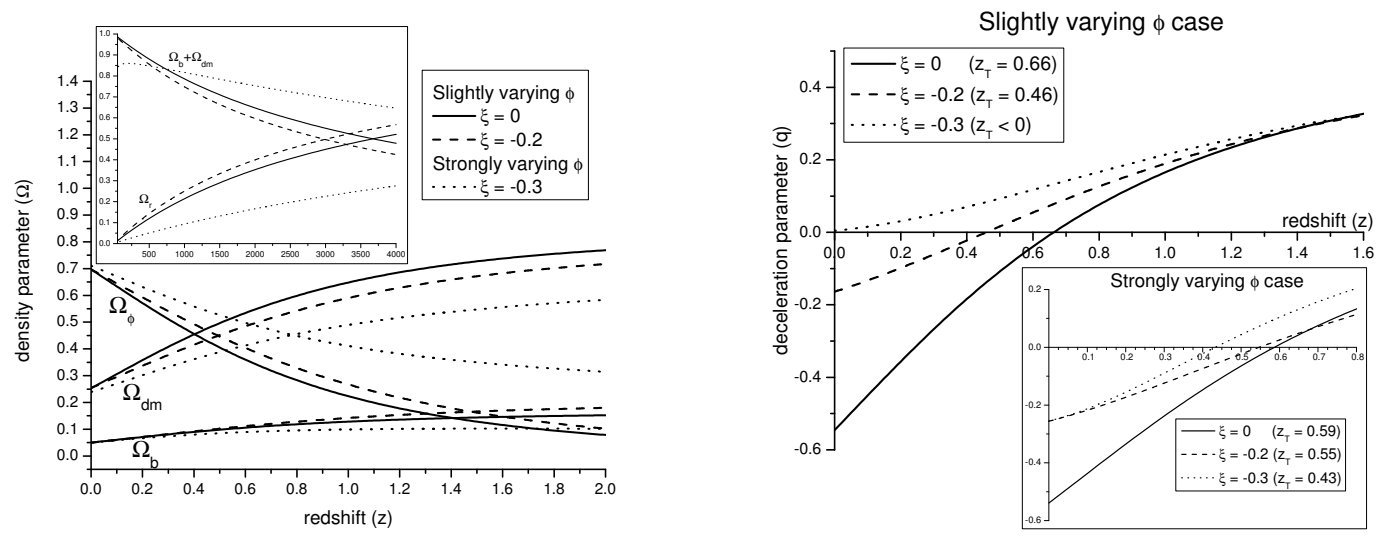

Figure 2: Left: evolution of density parameters $\Omega_{i}$ vs. red-shift $z$, showing that the coupling changes dark energy-dark matter equality and radiation-matter equality with the red-shift; right: deceleration parameter $q$ vs. red-shift $z$, the transition red-shift is closer to 0 as the coupling increases.

By changing the values of the potential exponent between the range $0 \leq \alpha \leq 5$ it is not possible to see any significant difference in the behavior of the curves in figure 2 however, for larger values $(\alpha \approx 10)$, there is a slower decay of the dark energy density and a later transition to the decelerated phase. This occurs because $V$ is of the same order as the other terms for red-shifts up to $z \approx 4$, and in this range the potential is mainly controlled by the value of $K$ for a slightly varying $\phi$.

In figure 3 we have plotted the difference $\mu_{0}$ between the apparent magnitude $m$ and the absolute magnitude $M$ of a source, given by $\mu_{0}=m-M=5 \log d_{L}+25$ with

$$
d_{L}=(1+z) c H_{0}^{-1} \int_{0}^{z} \frac{d z}{H(z)}
$$

as a function of the red-shift $z$, where $d_{L}$ is the luminosity distance given in Mpc. In this figure the circles represent the experimental values taken from the work by Riess et al. [5] for 185 data points of super-novae of type Ia, whereas the dash-dot line stands for the standard $\Lambda C D M$ model. We infer from the figure that all cases fit well at low red-shifts, but only the strongly varying case with $\xi=-0.3$ stays near the $\Lambda C D M$ over all the experimental range.

To summarize, the best solutions are those that allow the scalar field to change only until the dark energy density parameter becomes small and the scalar field slope tends to a small positive quantity.

\section{Dark Matter with non-vanishing pressure}

Let us analyze the case where the dark matter has a non-vanishing pressure. For this end we recall that in the presence of matter creation the first law of thermodynamics for adiabatic $(d Q=0)$ open systems reads 25]

$$
d Q=d(\rho V)+p d V-\frac{\rho_{d m}+p_{d m}}{n_{d m}} d\left(n_{d m} V\right)=0,
$$

where $V$ denotes the volume and it was supposed that only dark matter creation is allowed. By considering $V \propto a^{3}$ and the conservation laws for baryons and radiation it follows from (20)

$$
\dot{\rho}_{\phi}+\dot{\rho}_{d m}+3 H\left(\rho_{\phi}+\rho_{d m}+p_{\phi}+p_{d m}\right)-\frac{\rho_{d m}+p_{d m}}{n_{d m}}\left(\dot{n}_{d m}+3 H n_{d m}\right)=0 .
$$




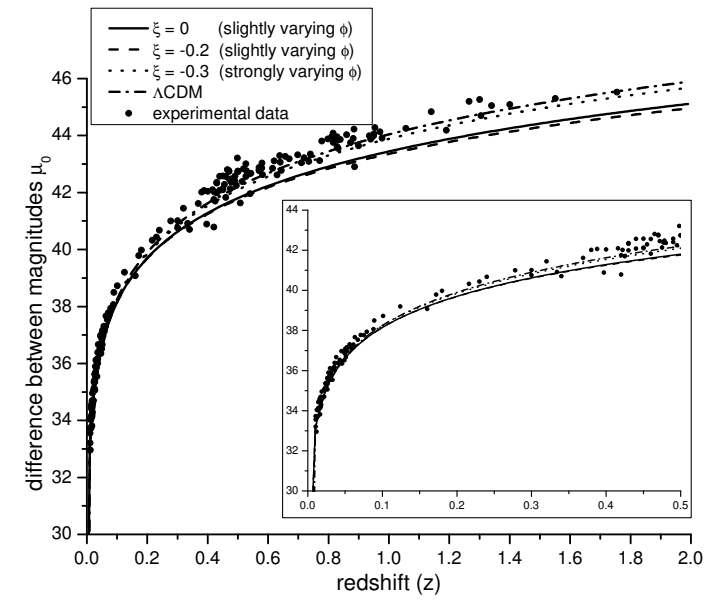

Figure 3: Difference $\mu_{0}$ vs. red-shift $z$, showing that coupling can increase or decrease the difference between magnitudes.

If we compare the above equation with the sum of equations (14) and (15), we obtain

$$
\frac{2 \xi \phi \dot{\phi} \rho}{1-\xi \phi^{2}}=-\frac{\rho_{d m}+p_{d m}}{n_{d m}}\left(\dot{n}_{d m}+3 H n_{d m}\right) .
$$

Now by using again (15) and $\rho_{d m}=m_{d m} n_{d m}=\zeta \phi n_{d m}$, we get the following expression for the pressure of the dark matter

$$
\frac{p_{d m}}{\rho_{d m}}=-\frac{1}{2} \pm \sqrt{\frac{1}{4}+\frac{2 \xi \phi \dot{\phi} \rho}{\left(1-\xi \phi^{2}\right)(3 H+\dot{\phi} / \phi) \rho_{d m}}} .
$$

We call attention to the fact that only the plus sign must be chosen in the above equation in order to obtain a positive dark matter pressure. Moreover, we observe that in this case, when $\xi=0$ we have from equation (22) that the particle number density of the dark matter is conserved, i.e., $\dot{n}_{d m}+3 H n_{d m}=0$ and from (23) that the dark matter pressure vanishes. For the case analyzed in previous section there exists no constraint in the values of $\xi$ when we assume from the beginning that the dark matter pressure vanishes.

We have now a system of coupled differential equations to solve, namely

$$
\begin{gathered}
\frac{(1+z)^{2} \rho}{3\left(1-\xi \phi^{2}\right)} \phi^{\prime \prime}-\frac{(1+z)(\rho-3 p)}{6\left(1-\xi \phi^{2}\right)} \phi^{\prime}+\frac{d V}{d \phi}+\frac{\xi \phi(\rho-3 p)}{1-\xi \phi^{2}}+\frac{\rho_{d m}-p_{d m}}{\phi}=0, \\
\rho_{d m}^{\prime}-\frac{3\left(\rho_{d m}+p_{d m}\right)}{(1+z)}=\left(\rho_{d m}-p_{d m}\right) \frac{\phi^{\prime}}{\phi}
\end{gathered}
$$

where $p_{d m}$ is given by (23) with the positive sign. The initial conditions used here are the same as those of the previous section.

Figure 4 corresponds to figure 2 for the case of a non-vanishing dark matter pressure, where we have analyzed two cases $(\xi= \pm 0.05)$ and compared them with the minimally coupled pressureless case. We observe from the left frame of this figure that the decay of the density parameter of the scalar field for the negative coupling constant is slower than the one for the pressureless case, whereas for the positive coupling constant the decay is more accentuated. This can also be understood by analyzing the term $-2 \xi \phi \dot{\phi} \rho /\left(1-\xi \phi^{2}\right)$ on the right-hand side of evolution equation for the energy density of the scalar field (14), since it changes its sign when $\xi$ is positive or negative and it is equal to zero for the pressureless case. However, for both cases the radiation-matter (baryons plus 

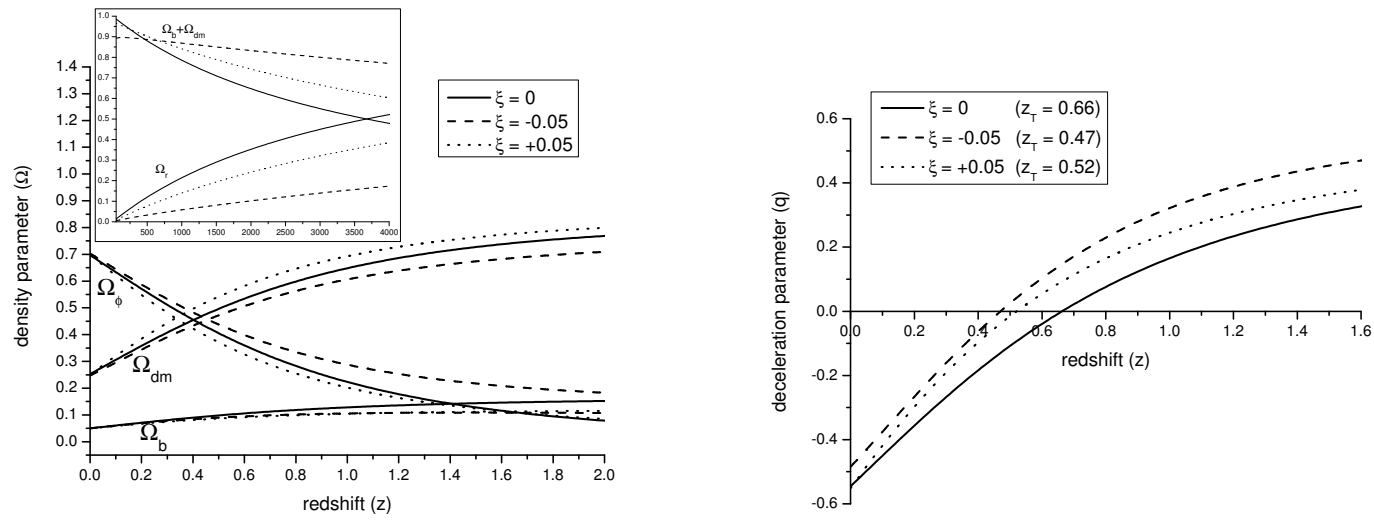

Figure 4: Left: evolution of density parameters $\Omega_{i}$ vs. red-shift $z$; right: deceleration parameter $q$ vs. red-shift $z$. The uncoupled case represents an intermediate case between positive and negative couplings for the density parameters at lower red-shifts $(z<2)$, and the coupling leads to a more recent transition redshift.

dark matter) equality occurs for higher red-shifts than that of the pressureless case. From the right frame of figure 4 we infer that the transition from a decelerated to an accelerated phase for $\xi=-0.05$ occurs at $z_{T}=0.47$ and the present deceleration parameter for this case approaches the value $q_{0} \approx-0.55$.

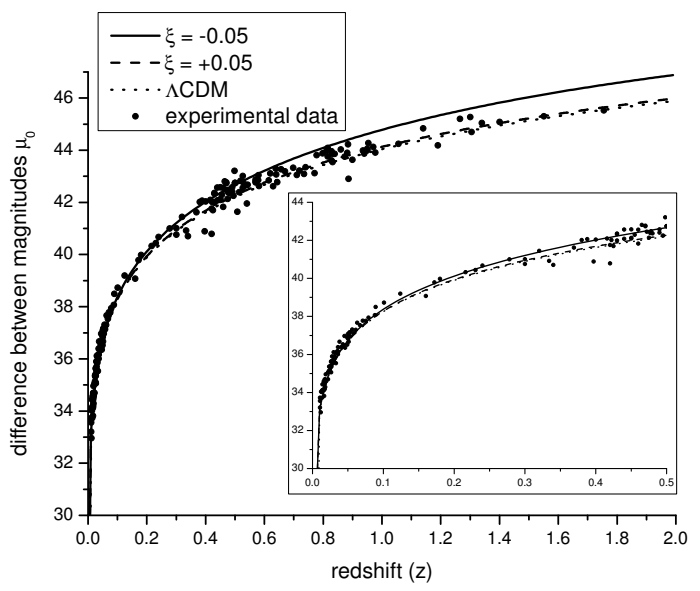

Figure 5: Difference $\mu_{0}$ vs. red-shift $z$ where the coupling may increase the difference between magnitudes.

In figure [ 5 it is plotted the difference between the apparent magnitude and the absolute magnitude of a source as a function of the red-shift for the case of a non-vanishing dark matter pressure. We observe that the $\xi=+0.05$ case stays near the $\Lambda C D M$ model, whereas the $\xi=-0.05$ case improves the behavior of the theoretical curve with respect to the experimental values for low red-shifts.

Finally, the left frame of figure [6 shows the evolution of the dark matter pressure for the situations discussed above. We note that for $\xi=-0.05$ the dark matter pressure vanishes when the red-shift goes to zero and tends to a small value for higher red-shifts. We emphasize that although low, the dark matter pressure introduced here has some significant influence on the behavior of the evolution of the whole system. For completeness we have plotted in the right frame of this figure 

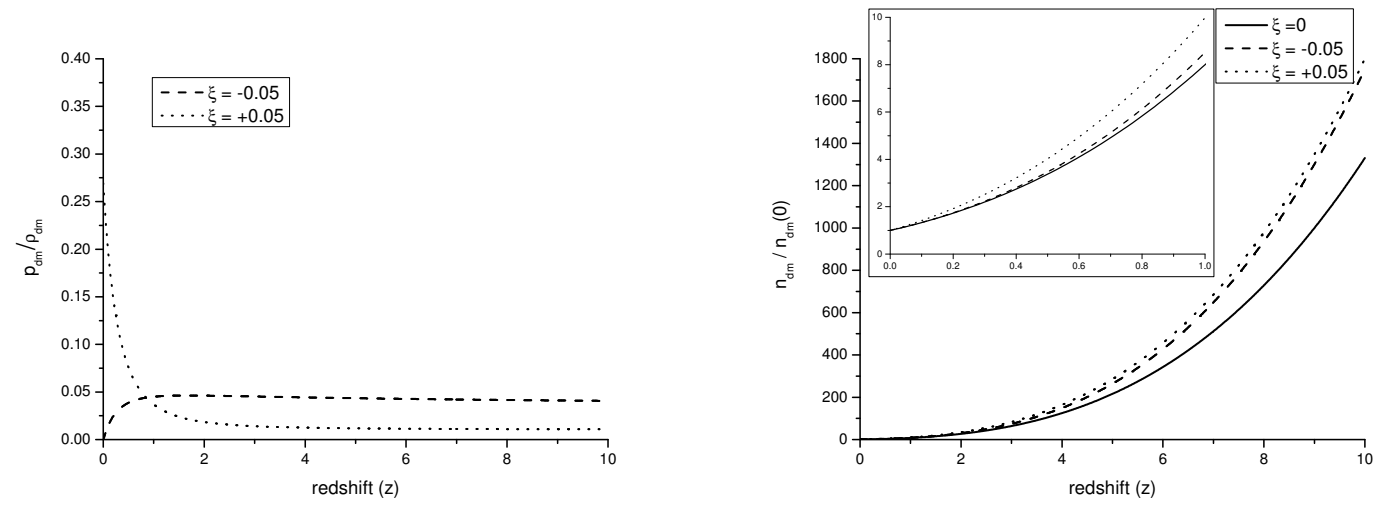

Figure 6: Left: dark matter pressure $p_{d m} / \rho_{d m}$ vs. red-shift $z$; right: dark matter particle number density $n_{d m}(z) / n_{d m}(0)$ vs. red-shift $z$, showing the influence of the coupling into the pressure and particle number density of the dark matter.

the evolution of the dark matter particle number density as a function of the red-shift, and, as was expected, the particle number densities for the non-vanishing pressure cases increase more rapidly with the red-shift than that of the pressureless case.

\section{$5 \quad$ Final remarks and conclusions}

An equivalent description for the model studied in previous sections is to write Einstein field equations as

$$
R_{\mu \nu}-\frac{1}{2} R g_{\mu \nu}=-\mathcal{T}_{\mu \nu}
$$

In this case, the energy-momentum tensor of the sources is conserved, i.e., $\nabla_{\nu} \mathcal{T}^{\mu \nu}=0$, whereas the energy-momentum tensor of the scalar field reads

$$
\mathcal{T}_{\mu \nu}^{\phi}=\partial_{\mu} \phi \partial_{\nu} \phi-\left(\frac{1}{2} \partial_{\sigma} \phi \partial^{\sigma} \phi-V\right) g_{\mu \nu}-\xi\left(\nabla_{\mu} \nabla_{\nu} \phi^{2}-g_{\mu \nu} \nabla_{\sigma} \nabla^{\sigma} \phi^{2}\right)+\xi \phi^{2}\left(R_{\mu \nu}-\frac{1}{2} R g_{\mu \nu}\right) .
$$

By considering the Robertson-Walker metric and the representation for the energy-momentum tensor $\left(\mathcal{T}^{\mu}{ }_{\nu}\right)=\operatorname{diag}(\tilde{\rho},-\tilde{p},-\tilde{p},-\tilde{p})$ it follows the Friedmann and acceleration equations in their usual forms, i.e.,

$$
H^{2}=\frac{\tilde{\rho}}{3}, \quad \frac{\ddot{a}}{a}=-\frac{\tilde{\rho}+3 \tilde{p}}{6} .
$$

However, the energy density and the pressure of the scalar field in this description have new contributions that are given by the underlined terms:

$$
\begin{gathered}
\tilde{\rho}_{\phi}=\frac{1}{2} \dot{\phi}^{2}+V+6 \xi H \phi \dot{\phi}+\underline{\xi \phi^{2} \tilde{\rho}}, \\
\tilde{p}_{\phi}=\frac{1}{2} \dot{\phi}^{2}-V-2 \xi\left(\phi \ddot{\phi}+\dot{\phi}^{2}+2 H \phi \dot{\phi}\right)+\underline{\xi \phi^{2} \tilde{p}},
\end{gathered}
$$

so that the sums $\tilde{\rho}=\tilde{\rho}_{\phi}+\rho_{d m}+\rho_{b}+\rho_{r}$ and $\tilde{p}=\tilde{p}_{\phi}+p_{d m}+p_{b}+p_{r}$ give

$$
\tilde{\rho}=\frac{\rho_{\phi}+\rho_{d m}+\rho_{b}+\rho_{r}}{1-\xi \phi^{2}}=\frac{\rho}{1-\xi \phi^{2}}, \quad \tilde{p}=\frac{p_{\phi}+p_{d m}+p_{b}+p_{r}}{1-\xi \phi^{2}}=\frac{p}{1-\xi \phi^{2}},
$$

showing the equivalence between this description and the former one. 
To summarize, we first observe that this model has a strong dependence on the initial conditions. For the pressureless dark matter case we have shown that: (a) a stronger coupling between the scalar field and the scalar curvature leads to a decelerated-accelerated transition and matter-radiation equality at lower red-shifts; (b) a stronger scalar field variation implies that stronger couplings adjust better with the experimental data of luminosity distance, but the matter-radiation equality occurs at higher red-shifts. For the case of a non-vanishing dark matter pressure we have shown that negative small values of the coupling constant leads to: (a) a decelerated-accelerated transition at lower red-shifts with a better adjustment of the present value of the deceleration parameter, but the

matter-radiation equality occurs at higher reds-shifts; (b) a better adjustment with the experimental data of luminosity distance at low red-shifts.

\section{Acknowledgment}

We are indebted to Prof. E. Gunzig for calling our attention to the case analyzed in the final comments.

\section{References}

[1] Persic, M., Salucci P., and Stel F. (1996). Mon. Not. Roy. Astron. Soc. 281, 27.

[2] Riess, A. G. et al, (1998). Astron. J. 116, 1009.

[3] Perlmutter, S. et al, (1999). Astrophys. J. 517, 565.

[4] Carroll, S. M. (2001). Liv. Rev. Rel. 4, 1.

[5] Riess, A. G. et al, (2004). Astrophys. J. 607, 665.

[6] Alcaniz, J. S. (2004). Phys. Rev. D 69, 083521.

[7] Carroll, S. M., Hoffman,M., and Trodden, M. (2003). Phys. Rev D 68, 023509.

[8] Hsu, S. D. H., Jenkins, A., and Wise, M. B. (2004). Phys. Lett. B 597, 270.

[9] Madsen, M. S. (1988). Class. Quant. Grav. 5, 627.

[10] Uzan, J. P. (1999). Phys. Rev. D 59, 123510.

[11] Chiba, T. (1999). Phys. Rev. D 60, 083508.

[12] Amendola, L. (1999). Phys. Rev. D 60, 043501.

[13] Esposito-Farèse G., and Polarski, D. (2001). Phys. Rev. D 63, 063504.

[14] Faraoni, V. (2001). Int. J. Theor. Phys. 40, 2259.

[15] Abramo, L. R., Brenig L., and Gunzig, E. (2002). Phys. Lett. B 549, 13.

[16] Casas, J. A., Garcia-Bellido J., and Quiros, M. (1992). Class. Quant. Grav. 9, 1371.

[17] Wetterich, C. (1995). Astron. Astrophys. 301, 321.

[18] Amendola, L. (2000). Phys. Rev. D 62, 043511.

[19] Alves D. S. M., and Kremer, G. M. (2004). JCAP 10, 009.

[20] Anderson G. W., and Carroll, S. M. (1997). astro-ph/9711288

[21] Hoffman, M. B. (2003). astro-ph/0307350 
[22] Liddle A. R., and Lyth, D. H. (2000). Cosmological Inflation and Large-Scale Structure, Cambridge University Press, Cambridge.

[23] Farrar G. R., and Peebles, P. J. E. (2004). Astrophys. J. 604, 1.

[24] Fukugita M., and Peebles, P. J. E. (2004). Astrophys. J. 616, 643.

[25] Prigogine, I., Géhéniau, J., Gunzig E., and Nardone, P. (1989). Gen. Rel. Grav. 21, 767. 